\title{
Size and Function
}

\author{
Bruno Whittle ${ }^{1}$ (iD
}

Received: 28 July 2016/ Accepted: 10 June 2017/Published online: 12 July 2017

(C) The Author(s) 2017. This article is an open access publication

\begin{abstract}
Are there different sizes of infinity? That is, are there infinite sets of different sizes? This is one of the most natural questions that one can ask about the infinite. But it is of course generally taken to be settled by mathematical results, such as Cantor's theorem, to the effect that there are infinite sets without bijections (i.e. one-to-one correspondences) between them. These results (which I of course do not dispute) settle the question, given an almost universally accepted principle relating size to the existence of functions. The principle is: for any sets $\mathrm{A}$ and $\mathrm{B}$, if $\mathrm{A}$ is the same size as B, then there is a bijection from A to B. The aim of the paper, however, is to argue that this question is in fact wide open: to argue that we are not in a position to know the answer, because we are not in one to know the principle. The aim, that is, is to argue that for all we know there is only one size of infinity.
\end{abstract}

\section{Introduction}

This paper is about a question that many readers will think has already been settled: are there different sizes of infinity? That is, are there infinite sets of different sizes? This is one of the most natural questions that one can ask about the infinite. But it is of course generally taken to be settled by mathematical results, such as Cantor's theorem, to the effect that there are infinite sets without bijections between them. ${ }^{1}$ An answer to our question is entailed by these results (which I of course do not

\footnotetext{
1 A bijection (or 'one-to-one correspondence') from $A$ to $B$ is a function that 'pairs' every member of $A$ with a distinct member of $B$, and vice versa. More precisely, let $f$ be a function from $A$ to $B . f$ is an injection from $A$ to $B$ if: for any $x, y \in A$, if $x \neq y$, then $f(x) \neq f(y)$. $f$ is a surjection from $A$ to $B$ if: for any
}

Bruno Whittle

bruno.whittle@glasgow.ac.uk

1 Department of Philosophy, University of Glasgow, Glasgow G12 8QQ, UK 
dispute), given the following almost universally accepted principle relating size to the existence of functions.

$\mathrm{Size} \rightarrow$ FunCTION

For any sets $A$ and $B$, if $A$ is the same size as $B$, then there is a bijection from $A$ to $B$.

For the results and the principle give us that there are infinite sets that are not of the same size, from which it would seem to follow that there are such sets of different sizes. The main aim of this paper, however, is to argue that this question is in fact wide open: to argue that we are not in a position to know the answer, because we are not in a position to know SizE $\rightarrow$ FunCtion. (By saying that we are not in a position to know something, I mean just that we don't know it, and there is no obvious way in which to remedy this fact.) The aim, that is, is to argue that for all we know there is only one size of infinity.

The standard modern account of size for sets consists of SizE $\rightarrow$ Function and its converse (together with related claims about when one set is smaller or larger than another).

SizE $\leftarrow$ Function

For any sets $A$ and $B, A$ is the same size as $B$, if there is a bijection from $A$ to $B$.

(I will also use SizE $\leftrightarrow$ Function for the biconditional claim.) However, while this converse has been the object of philosophical attention, SizE $\rightarrow$ Function has been surprisingly neglected. And this neglect is surprising, given that it is SizE $\rightarrow$ FunCTION, and not the converse, that is needed to derive that there are different sizes of infinity — which, after all, might reasonably be described as the most important claim of modern set theory. For example, in the opening pages of (1964), Gödel presents himself as arguing for the claim that $A$ has the same number of members as $B$ iff there is a bijection from $A$ to $B$ (which would seem to be a relatively minor variant of SizE $\leftrightarrow$ FunCTION). However, what he actually gives is essentially just an argument for SizE $\leftarrow$ Function, leaving Size $\rightarrow$ Function to fend for itself. Indeed, quite generally, arguments for SizE $\rightarrow$ FunCTION (or accounts of why we are entitled to believe it) are astonishingly thin on the ground. For example, while in Michael Hallett's magisterial treatment of Cantor's development of set theory we are told that Cantor's theory of cardinality is 'inevitable' (1984: xiv), the closest we get to an argument for this claim uses SIZE $\leftrightarrow$ FUNCTION as an unsupported premise (1984: 8889). Similarly, although Size $\leftarrow$ Function has been challenged (e.g. by Bolzano (1851), Parker (2009) and Mancosu (2009)), there is no comparable history of challenges to SizE $\rightarrow$ FunCTION. $^{2}$

Footnote 1 continued

$x \in B$, there is $y \in A$ with $f(y)=x$. $f$ is then a bijection from $A$ to $B$ if it is both an injection and a surjection from $A$ to $B$.

2 If SizE $\leftarrow$ Function could in fact be shown to be false, for example, then that would give us a route to knowledge of different sizes of infinity that would not go via SizE $\rightarrow$ FunCTION. I will take it, however, that arguments such as that given by Gödel do indeed show SizE $\leftarrow$ FunCtion to be true, and thus that we are in a position to know that there are different such sizes only if we are in one to know SizE $\rightarrow$ FunCTION. 
This lack of attention is even more surprising given how much discussion there has in recent years been of Hume's principle (which, again, would seem to be a minor variant of SIZE $\leftrightarrow$ FUNCTION): ${ }^{3}$

$$
\forall F \forall G(\# F=\# G \leftrightarrow F \approx G) .
$$

Indeed, when in his seminal (1983) Crispin Wright comes to consider the question of why we should in fact believe this, and, in particular, why we should believe the left-to-right direction of it (i.e. SizE $\rightarrow$ FunCtion), what we are given is simply an argument that he says works in the finite case. Why believe that it also holds in the infinite one? 'Good question', he in effect says (1983: 107). But-actually-if the arguments below are correct then that would seem about right. For although I will focus on claims about size rather than number, it would seem to follow from these that just as we are not in a position to know SizE $\rightarrow$ Function, neither are we in one to know (the left-to-right direction of) Hume's principle.

\subsection{Scepticism}

Some readers, however, will already be sceptical. 'Surely' - they will think-'there being a bijection from $A$ to $B$ is simply what it is for $A$ to be the same size as $B$ (and so of course we are in a position to know SizE $\rightarrow$ Function)' ${ }^{4}$ Thus, on the most straightforward way of understanding this, the suggestion is that ' $c$ is the same size as $d$ ' expresses the very same proposition as 'there is a bijection from $c$ to $d$ '. ${ }^{5}$ But if that is the case, then SizE $\rightarrow$ Function would seem to be a proposition of the form $\langle$ for any sets $A$ and $B$, if $p$, then $p\rangle$-which surely is something that are in a position to know! ${ }^{6}$

On reflection, however, it just doesn't seem plausible that there is anything like such a strong connection between these sentences. For prima facie ' $c$ is the same size as $d$ ' expresses a proposition to the effect that there is a certain sort of property-a size - that $c$ and $d$ have in common:

$$
\begin{aligned}
& \langle\exists ! P(\operatorname{Size}(P) \wedge c \text { has } P) \wedge \exists ! Q(\operatorname{Size}(Q) \wedge d \text { has } Q) \wedge \exists R(\operatorname{Size}(R) \wedge c \text { has } \\
& R \wedge d \text { has } \mathrm{R})\rangle .
\end{aligned}
$$

In contrast, 'there is a bijection from $c$ to $d$ ' would seem to express a proposition about a very different sort of thing - a function:

\footnotetext{
${ }^{3}$ Here $F$ and $G$ range over Fregean concepts, $\# F$ means the number of $F$ s, and $F \approx G$ means that there is a bijection from the $F$ s to the $G$ s.

4 A closely related thought: what it is for $A$ to be the same size as $B$ is for it to be possible to 'count' their members and arrive at the same result. However, everything that I will say can easily be adapted to this alternative.

5 Less straightforward ways of understanding the remark, e.g. in terms of 'reconceptualizing' or 'describing the same features of reality', will be considered in Sect. 2 below.

${ }^{6}$ I use angle brackets to refer to propositions. Unless otherwise stated, I for simplicity assume a Russellian account of these. However, everything that I will say could be adapted to any alternative account, such Fregean or possible worlds accounts: see, e.g., later in this subsection.
} 
$\langle\exists f f$ is a bijection from $c$ to $d\rangle$.

If these sentences in fact express the same proposition, then presumably only one of these appearances is correct, i.e. either both express (1) or both express (2).

Neither proposal seems promising, however. For surely most sentences about functions express propositions that are genuinely about these. For example, consider 'there is a constant function from $c$ to $d$ '. There does not seem to be any plausible account of this on which it in fact expresses a proposition about properties. But it beggars belief to think that such apparently similar sentences in fact express propositions about completely different sorts of things. Thus it seems that 'there is a bijection from $c$ to $d$ ' cannot express (1). On the other hand, however, ' $c$ is the same size as $d$ ' is a sentence of the same general form as ' $e$ is the same colour as $g$ ', ' $e$ is the same height as $g$ ', ' $e$ is the same sex as $g$ ' etc. And it is surely very plausible that these sentences also express propositions of the same general form. But it is clear that the propositions that these other sentences express are not about functions. For example, there does not seem to be any way of thinking of ' $e$ is the same colour as $g$ ' as in fact asserting the existence of a bijection. But then it seems that ' $c$ is the same size as $d$ ' cannot express (2). That is, it seems that the sentences do not express the same proposition.

What if we instead identify propositions with sets of possible worlds? On this account, ' $c$ is the same size as $d$ ' and 'there is a bijection from $c$ to $d$ ' express the same proposition iff they are necessarily equivalent; which is presumably the case iff SizE $\leftrightarrow$ FunCTION is true. In this case, there is no quick refutation of the claim that they do in fact express the same proposition. By the same token, however, the fact that they do express the same proposition - assuming for a moment that it is a factwould be of little help in providing an easy route to the claim that we know Size $\rightarrow$ Function. For on this account of propositions, the fact that $\alpha$ and $\beta$ express the same proposition certainly does not mean that we are in a position to know $\alpha \rightarrow \beta$ (or generalizations of this); otherwise we would be in a position to decide every pure mathematical claim $\gamma$ whatsoever (since we would be in a position to know either $0=0 \rightarrow \gamma$ or $0=0 \rightarrow \neg \gamma$, and hence either $\gamma$ or $\neg \gamma$ ). An easy route to knowledge of SizE $\rightarrow$ FUNCTION — akin to that considered above in terms of Russellian propositions-would within this framework thus require something quite different. For example, such a route might be afforded by the claim that our two sentences express the same propositions via the same 'structured meanings'. But the considerations given above would seem to show that the sentences do not have the same meanings in this sense. And similarly, it would seem, for other attempts to find an easy route to knowledge of SizE $\rightarrow$ Function. On either conception of proposition, then, this sort of scepticism about the project is misplaced.

\subsection{Indeterminacy}

The target of the paper is the claim that we are in a position to know SizE $\rightarrow$ FunCtion. This would seem to require that the ordinary, intuitive notion of size is sufficiently determinate to at least fix the truth-value of SizE $\rightarrow$ Function. So this is an assumption that I will make for most of the paper, at least for the sake of 
argument. However, there is a very different way of thinking about these things that some readers might be tempted by. According to this, while we do-in a senseknow that there are different sizes of infinity, the ordinary notion of size is not in fact so determinate. Thus we do not know Size $\rightarrow$ Function. What we do know, however, is the next best thing, i.e. the following.

SizE $\rightarrow$ FunCTION

There is some 'size-like' property size* such that:

for any sets $A$ and $B$, if $A$ is the same size* as $B$, then there is a bijection from $A$ to $B$.

That is, there is some property size* that is picked out by a notion that is at least very similar to that of size (e.g. a sharpening of that notion), and that satisfies the conditional in question. We would then be in a position to know that there are different sizes of infinity at least in the sense of knowing that there are different such 'sizes*'. For example, one version of this view might maintain that set theory provides us with a 'size-like' property in this sense. Indeed, even if SizE $\rightarrow$ FunCTION is determinately false, for example, one might think that knowledge of SIZE $\rightarrow$ FunCTION would still in some important way vindicate the claim that we know that there are different sizes of infinity. In fact, however, although the main focus will be on SIZE $\rightarrow$ Function, the arguments that I will give against the claim that we are in a position know this would seem to extend even to the weaker one that we are in a position to know SizE* $\rightarrow$ Function (see Sect. 9). ${ }^{7}$

Nevertheless, since I will for most of the paper assume that our ordinary notion of size is sufficiently determinate to fix the truth-value of SizE $\rightarrow$ Function, I should say at least something to defend this claim; specifically, from what might seem to be the main threat to it, which is as follows. Thus one might worry that the ordinary notion of size is susceptible to paradox when we extend it to infinite sets, and that, as a result, claims such as SIZE $\rightarrow$ FunCTION lack a determinate truth-value. This worry would be based on the fact that although (as I have noted) there seem to be strong arguments for the converse principle SizE $\leftarrow$ Function, there also seem to be such arguments against it: in particular, those stemming from the fact that it is inconsistent with the 'part-whole principle', according to which a set is always bigger than its proper subsets. The ordinary notion of size might thus-like the ordinary notions of collection or truth-seem to be inconsistent, and so unsuitable for serious theorizing. The latter, it would be argued, requires rather some replacement, such as that offered by modern set theory.

This is not of course the place for a full discussion of this issue. ${ }^{8}$ I should however note that there seem to be strong prima facie grounds for questioning this line of thought - for there seem to be independent grounds to doubt the part-whole principle. But, in that case, there is every reason to favour SizE $\leftarrow$ Function over this principle, and so every reason to think that the ordinary notion is consistent (and sufficiently determinate for our purposes). Thus, consider $E$, the set of even numbers

\footnotetext{
7 Readers who think that, even if we might be able to challenge SizE $\rightarrow$ Function, SizE $\rightarrow$ Function is just obvious are asked to bear with me until Sect. 4, where I will argue against such judgements.

${ }^{8}$ For more extensive consideration of it, see Parker (2009) and Mancosu (2009).
} 
$0,2,4, \ldots$, and $O$, the set of odd ones $1,3,5, \ldots$ Even if we are uncertain about the general claim SizE $\leftarrow$ Function, we are surely going to want to say that $E$ and $O$ are the same size. However, the relationship that $E$ stands in to $O$ is precisely that which $O$ stands in to $E^{+}=E-\{0\}$. But then $E$ and $E^{+}$must be the same size too, in which case the part-whole principle fails. There is, of course, more to be said here. But these initial considerations do seem to go some way towards undermining what might have seemed to be the strongest grounds for doubting our assumption (i.e. about the determinacy of the ordinary notion of size); and thus some way towards defending that assumption.

\subsection{Interest}

Why, though, should we care whether there are different sizes of infinity? The main reason is simply that sizes are among the most natural and fundamental properties that sets have. Thus, the question of whether there is one such property that applies to every infinite set, or different ones that apply to different such sets, would seem to be among the most natural that one can ask about sets or about infinity. Further, it is clear that we $d o$ in fact care about this question. Why else would practically every introduction to set theory in existence present the theory's purported ability to answer this as one of its main selling points-if not its main one?

\subsection{Ontology}

The focus of this paper is a principle about sets and functions. Alternative versions of the principle would replace sets with Fregean concepts, properties or pluralities, and everything that I will say would apply to such alternatives. I focus on the principle about sets simply because these are most familiar, and least likely to lead to distracting controversies.

I will not take a stand on what exactly functions are. For everything that I will say would seem to be compatible with any reasonable account of this. For example, it will be compatible with their being sets of ordered pairs, but equally with their being sui generis objects, or some sort of 'higher-order' entity.

Similarly, I will not take a stand on the metaphysical status of either sets or functions. Rather, what I say will be compatible with their existing in the same sort of way that the objects of fundamental physics do, but also with their existing in some much more 'lightweight' manner-or even not existing at all, as long as good sense can be made of talk about them.

\subsection{Structure}

The main task of the paper, then, is to consider what seem to be the strongest reasons for thinking that we know (or are in a position to know) SizE $\rightarrow$ Function, and to argue against them. In Sect. 1.1 I considered the suggestion that there being a bijection from $A$ to $B$ is what it is for $A$ to be the same size as $B$, understood as the suggestion that ' $c$ is the same size as $d$ ' and 'there is a bijection from $c$ to $d$ ' in fact 
express the same proposition. We saw that this cannot be right. But one might still suspect that something close to it is. Specifically, one might suspect that the properties that the former sentence is about are themselves to be understood in terms of functions (so the sentence would indirectly be about functions). Further, as long as we know such a functional account of size, we would be in a position to know Size $\rightarrow$ Function. But in Sect. 2 we will see that such accounts are incorrect: sizes are rather intrinsic properties of sets (i.e. properties that sets have purely in virtue of what they are like, rather than which functions to or from the set there are). In Sect. 3 I will then consider the possibility of arguing for SizE $\rightarrow$ FunCTION on the basis that it holds in the finite case. In Sect. 4 I will take a break from considering arguments for Size $\rightarrow$ Function and consider SizE* $\rightarrow$ Function. Specifically, I will argue against the judgement that even if we might be able to challenge Size $\rightarrow$ Function, SizE $\rightarrow$ FunCTION is surely just obvious.

In Sect. 5 I will then consider what might seem to be the strongest argument for SizE $\rightarrow$ FunCtion, which is as follows. ${ }^{9}$ Consider a simple pair of finite sets, such as $C=\{0,1\}$ and $D=\{2,3,4\}$. Two things are true of these: (a) they are of different sizes, and (b) there is no bijection between them. But these things also seem to be connected: (a) seems to be the reason for-or the explanation of-(b). After all, if we are asked, 'Why is there no bijection between $C$ and $D$ ?', it is very natural to answer, 'Because they are of different sizes' (or 'Because $C$ is smaller than $D$ '). (In contrast, if we are asked, 'Why is $C$ a different size to $D$ ?', then it is not at all natural to reply, 'Because there is no bijection from $C$ to $D$ '.) But it is then tempting to think that this must always be how things are. That is, that for any sets $A$ and $B$, if there is no bijection between them, then this fact must similarly have an explanation, and further that the only one possible is in terms of size (i.e. the sets differ in size). But then Size $\rightarrow$ Function must hold. Indeed, I would suggest that some version of this argument is at least one of the reasons that we find Size $\rightarrow$ Function so appealing. I will argue, however, that it fails. Specifically, I will argue that in the paradigm cases of infinite sets that we know not to have bijections between them, such as the set of natural numbers $\mathbb{N}$ and its powerset $\mathcal{P}(\mathbb{N})$, there is actually an alternative explanation of this fact that is available. ${ }^{10}$ This alternative (straightforwardly inspired by a standard argument for Cantor's theorem) replaces talk of size with a principle to the effect that for any function $f$ from $\mathbb{N}$ to $\mathcal{P}(\mathbb{N})$, there is a 'diagonal' set $D_{f}$, whose construction guarantees that it must be outside the range of $f$. This principle, I will argue, allows for a completely 'sizeless' explanation of the fact that there is no bijection from $\mathbb{N}$ to $\mathcal{P}(\mathbb{N})$.

Thus, although the main concern of the paper is the epistemic status of SizE $\rightarrow$ FunCTION (and hence of the claim that there are different sizes of infinity), we will also arrive at some metaphysical results. In particular, a new picture of why certain fundamental claims about sets and functions (e.g. claims to the effect that there is no bijection between a given pair of sets) are true. This will show that a range of thoughts that might initially seem obvious must in fact be given up.

\footnotetext{
9 The discussion of this section is a significantly expanded version of that of Whittle (2015: 11-15).

${ }^{10}$ If $A$ is a set, then its powerset, $\mathcal{P}(A)$, is the set of its subsets.
} 
In Sect. 6 I will then consider the finite case of SizE $\rightarrow$ Function. I will point out that while there is a strong argument for this, it will not extend to the general case. Having seen that the apparently most promising strategies for arguing for Size $\rightarrow$ Function fail, I will in Sect. 7 consider the possibility that this principle is in fact sufficiently obvious that we are entitled to treat it as basic, i.e. believe it even in the absence of such an argument. The reason for waiting so long before considering this idea is just that the considerations of preceding sections will be helpful in making clear that SizE $\rightarrow$ FunCTION is not obvious. In Sect. 8 I will consider the possibility of giving an inductive argument for the principle, i.e. an argument based on the virtues of the mathematical or philosophical results that it lets us derive. In Sect. 9 I will then come back to SizE* $\rightarrow$ Function, explaining how the arguments of the preceding sections would seem to extend even to the weaker claim that we are in a position to know this. In conclusion I will consider some directions for future work.

\section{Functional Accounts of Size}

In this section, then, I will consider accounts of size in terms of functions. Such an account would seem to be next best thing to ' $c$ is the same size as $d$ ' expressing $\langle\exists f f$ is a bijection from $c$ to $d\rangle$. For on such an account the proposition expressed by this sentence would at least indirectly be about functions.

On the prima facie most promising such account, sizes would be properties of the following form: ${ }^{11}$

$\langle$ there is a bijection from _ to $\kappa\rangle$,

where $\kappa$ is a set of some privileged sort, such as a cardinal (as defined in standard set theory). If these are what sizes are, then ' $c$ is the same size as $d$ ' would express something close to the proposition that there is a cardinal that $c$ and $d$ both have bijections to, which is in turn close to that to the effect that there is a bijection from $c$ to $d$. Further, such an account will straightforwardly entail SizE $\rightarrow$ FunCTION, and so if we know the account we would be in a position to know the principle. It seems, however, that no such account can be correct.

The most straightforward way in which to see this is as follows. Consider the sets $\{0,1\}$ and $\{2,3\}$, and a situation that is just like the actual world, except that there are no functions from either set. What would be true in this situation? It is, perhaps, metaphysically impossible. But, as we will see, we seem to be quite capable of judging what would be true in it (at least in the cases that I will be concerned with). And, after all, we consider metaphysically impossible situations in both philosophy and mathematics all the time. For example, it is plausibly either metaphysically impossible that there are sets, or metaphysically impossible that there are not. But we seem to have no difficulty in considering each hypothesis, and judging would be

${ }^{11}$ I assume that logically complex properties are Russellian propositions with 'gaps' or 'variables' in them, and so I refer to these too with angle brackets. As with propositions, however, it would seem possible to adapt anything that I will say to any alternative account. 
true under it. Similarly, in mathematics, when we prove by reductio that there is no surjection from $\mathbb{N}$ to $\mathcal{P}(\mathbb{N})$, we consider the metaphysically impossible hypothesis that there is such a function. And we have no difficulty at all in discerning what is, and what is not, an immediate consequence of this hypothesis. (Further, although the argument to follow is in terms of what is true under a metaphysically impossible hypothesis, one could, if one wanted to tighten the connection to standard reductio arguments, give a similar argument in terms of what is an immediate consequence of this hypothesis.) There should be little concern, then, about the legitimacy of this use of a metaphysically impossible hypothesis.

What would be true in the situation that I described? One thing that would clearly be true is that there would be no bijection from either $\{0,1\}$ or $\{2,3\}$. Thus, neither set would have a property of the form 〈there is a bijection from _ to $\kappa\rangle$ (for $\kappa$ a cardinal or anything else). On the other hand, it seems equally clear that the sets would still be of the same size. We surely wouldn't describe this as a situation in which $\{0,1\}$ is no longer the same size as $\{2,3\}$. But then the above account of size (in terms of bijections and cardinals) must be wrong: for if it was right, this would be a situation in which neither set has a size at all (since neither has a property of the form in question), and so they could not have the same one.

Further, a similar argument would seem to be available to show that any alternative functional account must also fail. Indeed, arguments along these lines would seem to show that any account of the size of a set in terms of things that are extrinsic to that set must similarly fail. (By saying that something is extrinsic to a set, I mean that it is not one of the things that constitute that set, such as its members, their parts or their members.) That is, sizes seem to be intrinsic properties of sets.

\section{1 'What It Is'}

In Sect. 1.1 I considered the idea that there being a bijection from $A$ to $B$ is what it is for $A$ to be the same size as $B$, understanding this as requiring that ' $c$ is the same size as $d$ ' and 'there is a bijection from $c$ to $d$ ' express the very same proposition. As I noted, however, alternative understandings would also seem to be available. For example, an understanding on which the proposition expressed by the sentence about size (i.e. (1)) is a 'reconceptualization' of that expressed by the sentence about bijections (i.e. (2)), or on which the two propositions 'describe the same feature of reality'. ${ }^{12}$ However, these alternatives would also seem to be refuted by the thought experiment just considered. For if (1) is a reconceptualization of - i.e. just another way of saying - (2), then given that (2) is false in the situation described, (1) should be false too. But we have seen that (1) is in fact true in this situation. Similarly, if the two propositions describe the very same feature of reality, then, given that (2) is false in the situation, one would again expect (1) to be too. It seems, therefore, that these alternative understandings are incorrect, just as the more straightforward one is. ${ }^{13}$

\footnotetext{
12 For this notion of reconceptualization, see Wright (1997), for that of describing the same feature of reality, see Rayo (2013).

13 Am I dismissing the whole neo-Fregean tradition (i.e. that begun by Wright (1983)) on the basis of the above thought experiment? Certainly not. The thought experiment does seem to show that claims involving the ordinary notion of number-just like those involving the ordinary notion of size-are far
} 


\subsection{Conceptions of Function}

One might worry that this is a point where our conception of function makes a difference. For suppose that we think of these as Fregean concepts, for example. Then a situation in which there is no bijection from $\{0,1\}$ to $\{2,3\}$ will not just be metaphysically impossible, but logically so (at least arguably). Even if we can make sense of metaphysically impossible situations, can we really make sense of logically impossible ones? In fact, however, when it comes to this branch of logic (and so this type of logical impossibility) we seem to have no difficulty at all. For just as we can make sense both of the hypothesis that there are sets, and also the hypothesis that there are not, so we can make sense both of the hypothesis that there are Fregean concepts, and also that to the effect that there are not. Similarly, we have no difficulty at all reasoning on the basis of the hypothesis that there is a Fregean concept that is a surjection from $\mathbb{N}$ to $\mathcal{P}(\mathbb{N})$ (e.g. in the course of proving that there is no such concept). It seems, then, that the argument above succeeds even if we think of functions in such a 'higher-order' way. ${ }^{14}$

\subsection{A Further Problem}

What I have said would seem to be quite sufficient to see off functional accounts of size. Nevertheless, it is worth pointing out that there is a further problem that the specific account considered above (i.e. that in terms of bijections and cardinals) suffers from. This is a version of the 'Benacerraf problem' (see (1965)), which afflicts identifications of the natural numbers with sets (e.g. $\varnothing,\{\varnothing\},\{\{\varnothing\}\} \ldots$ or $\varnothing,\{\varnothing\},\{\varnothing,\{\varnothing\}\} \ldots)$. The original problem is simply that any such identification would seem arbitrary, and thus incorrect. But the account of size in terms of cardinals would seem to face a similar problem. For the way in which cardinals are defined is as follows. One first defines ordinals (i.e. $\varnothing,\{\varnothing\},\{\varnothing,\{\varnothing\}\}$ and so on), and then cardinals as distinguished ones of these. But there are other families of sets

Footnote 13 continued

from reconceptualizations of claims about bijections. However, this seems quite compatible with the main claims of the neo-Fregean tradition (for which see, e.g., the introduction of Hale and Wright (2001)). These central claims require only that knowledge of the existence of abstract objects can be grounded in analytic 'abstraction principles', such as Hume's principle. They do not require that these principles are analytic of our ordinary notions. After all, Frege's original programme, quite apart from the trouble with Basic Law V, would seem untenable as an account of the ordinary notion of number, for example. Since Frege identified numbers with sets (or extensions). But the considerations of Benacerraf (1965) would seem to show that, when understood in terms of the ordinary notion, no such identification is correct (see Sect. 2.3 below). Of course, the neo-Fregean will want claims about their notion of number to be necessarily equivalent to claims about the ordinary notion-something that the conclusion of this paper would seem to challenge, at least in the sense of challenging our claim to know this. But this conclusion is certainly not based on the above thought experiment alone.

14 There is a terminological point that I should flag, however. I have been assuming that if sizes are intrinsic, then they do not involve functions. But one might insist that an intrinsic property is simply one that applies to an object independently of its relations to other objects, in which case sizes could be intrinsic while still involving functions, as long as these are taken to be Fregean concepts. Nothing for these purposes turns on this point, however. If this is the right way to use 'intrinsic', then my claims must just be reformulated using a stronger notion, that rules out dependence on functions, however these are understood. 
that one could apparently just as well characterize sizes in terms of. For example, rather than thinking of ordinals as sets in this way one could instead think of them essentially as we do natural numbers, i.e. as a sui generis family of objects without anything like members. Call such an object an ordinal*. One could then easily define a notion of cardinal in terms of these: for any ordinal* $\lambda$, let $\lambda^{*}$ be the set of ordinals that come before $\lambda$; a cardinal $*$ would then be an ordinal* $\kappa$ such that for no earlier $\lambda$ is there a bijection from $\lambda^{*}$ to $\kappa^{*}$. A characterization of size in terms of cardinal*s would then seem just as apt as that in terms of cardinals: i.e. sizes would be properties of the form $\left\langle\right.$ there is a bijection from _ to $\left.\kappa^{*}\right\rangle$ for some cardinal* $\kappa$. Thus - just as with natural numbers and sets - the identification of sizes with either of these families of properties would seem arbitrary, and thus incorrect.

The upshot of this section is that functional accounts of size are untenable. We should be clear, however, that it certainly does not yet follow that there is anything wrong with using functional properties to investigate size (since sizes may still be coextensive with such properties). For example, it certainly does not yet follow that there is anything wrong with the standard mathematical definition of cardinality - as long as this is not taken to be an account of what sizes are. This definition will be useful in connection with investigating size, as long as SizE $\rightarrow$ Function is true (something that we are of course yet to rule out). What does follow from the above arguments is simply that-contrary to what we might have hoped-we cannot establish that this principle is in fact true via a functional account of size. ${ }^{15}$

\section{Arguing from the Finite Case}

Without question, the finite case of SizE $\rightarrow$ Function is true. I will consider how we know this in Sect. 6. But what about arguing for the general case just on the basis of this special one? The problem is that many things true of finite sets are not true generally. For example: if $A \subset B$ then $A$ is smaller than $B$; there is no injection from $A$ into a proper subset of $A$; if $A$ is a set of natural numbers, then there is some Turing machine that outputs precisely the members of $A$. But then the fact that something is true of finite sets is at best very weak evidence for its holding generally. Thus it seems that we cannot argue for the general case of the principle just on the basis of the finite one.

\footnotetext{
15 Rather than giving such a functional account of size, i.e. of the properties, one might instead give such an account of the notion, or concept, of size. For example, one might propose that this concept is: is $a$ property that applies exactly to the members of some bijection-type (or perhaps: is the most natural such property). Again, however, the thought experiment we considered would seem to refute such accounts. For in the situation described there is no bijection between $\{0,1\}$ and $\{2,3\}$, and so they do not share a bijection-type. Thus, they do not share a property applying exactly to the members of such a type. So on the proposal under consideration, ' $\{0,1\}$ is the same size as $\{2,3\}$ ' would be false in this situation. However, as we have in effect noted, this is in fact true in the situation. Alternative such accounts are possible, of course, but they seem to be defeated by similar arguments.
} 


\section{4 'Size-Like' Properties}

Suppose that, as I am in the process of arguing, we are not in fact in a position to know Size $\rightarrow$ Function. Nevertheless, one might still think that our claim to knowledge of different sizes of infinity would be vindicated-at least in some important sense-if we are in a position to know that there is some 'size-like' property that satisfies this principle. That is, that there is some property that is very similar to size that satisfies it. (Recall that I use SizE* $\rightarrow$ Function for this claim.) Indeed, I imagine that many readers, at least at the outset, will have thought that even if it might be possible to challenge Size $\rightarrow$ Function, SizE* $\rightarrow$ Function is surely just obvious. In fact, however, what we have seen should already make us doubt this judgement.

For why think that SizE $\rightarrow$ FunCTION is obvious? The reason is presumably something like this: in modern set theory we study a family of properties that satisfy Size $\rightarrow$ Function, i.e. powers or what are usually called 'cardinalities', which are properties of the form 〈there is a bijection from _ to $\kappa\rangle$ (for a cardinal $\kappa$ ). Surelyone might have thought-even if these are not in fact sizes, they are at least very similar to them. But then SizE $\rightarrow$ Function is true. One way of putting such a thought would be to say that the property of being a power is at least one natural generalization of that of being a finite size. However, plausible as such thoughts might initially have appeared, they seem just to be mistaken. For, firstly, the property of being a power isn't a generalization of that of a finite size: since the collection of powers doesn't include the finite sizes (as we saw in Sect. 2). Further, powers are just a completely different sort of property from sizes. For, unlike powers, sizes are intrinsic and do not have anything particularly to do with functions or cardinals. It is true, of course, that every finite size is coextensive with a power, but that certainly doesn't show that there is any real similarity between the two. Consider an analogy: suppose that for some set $M$ of masses, there is a measuring device $d$ (e.g. an electronic scale) such that for any objects $x$ and $y$ whose masses are in $M, x$ has the same mass as $y$ iff $x$ produces the same reading as $y$ when placed on $d$. It would certainly be a mistake to conclude from this that there is any real similarity between masses and properties of the form $\left\langle_{-}\right.$produces reading $r$ on $\left.d\right\rangle$ precisely because masses do not have anything particularly to do with measuring devices or readings on them. But the situation with sizes and powers seems in all essential respects to be the same: that is, it seems similarly to be the case that there is no real similarity between sizes and powers precisely because the former do not have anything particularly to do with functions or cardinals. ${ }^{16}$ It seems, then, that we should question those initially plausible judgements to the effect that SizE $\rightarrow$ FunCTION is just obvious.

\footnotetext{
16 If one is troubled by the fact that masses in $M$ will only be contingently coextensive with properties about readings on $d$ then just consider rigidified versions of these. That is, properties of having a certain mass in the actual world vs properties of producing a certain reading on $d$ in this world. These will be necessarily coextensive (given our hypothesis). But it is surely just as clear that there is no real similarity between these two sorts of properties.
} 


\section{An Explanatory Connection?}

We now come to what might seem the prima facie most compelling argument for SizE $\rightarrow$ Function.

\subsection{The Argument}

Thus, consider a simple pair of finite sets, such as $C=\{0,1\}$ and $D=\{2,3,4\}$. Two things are true of these: (a) they are of different size; and (b) there is no bijection from $C$ to $D$. But these things also seem to be connected: (a) seems to be the reason for-or the explanation of-(b). For if we are asked, 'Why is there no bijection from $C$ to $D$ ?', it is very natural to answer, 'Because $C$ is a different size from $D$ ' (or 'Because $C$ is smaller than $D$ '). In contrast, if we are asked, 'Why is $C$ a different size to $D$ ?', then it is certainly not natural to reply, 'Because there is no bijection from $C$ to $D$ '. Rather, one would say something about the members of $C$ and $D$, such as 'Because the only members of $C$ are 0 and 1 , whereas $D$ contains 2, 3 and 4 (all of which are distinct)'.

The explanation of why there is no bijection from $C$ to $D$ thus seems to be as follows, where an arrow from one fact to another means that the latter holds because the former does. (Call this explanation (E1).)

There is no bijection from $C$ to $D$

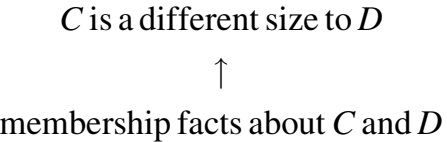

But it is then tempting to think that this must always be how things are. That is, that for any sets $A$ and $B$, if there is no bijection from $A$ to $B$, then there must similarly be some explanation of this fact, and further that the only one possible is that $A$ is a different size to $B$. But then of course SizE $\rightarrow$ FunCtion must hold. ${ }^{17}$

How plausible this line of thought can seem can be made even clearer by considering other, closely related ones. Consider the following principle, for example.

(3) For any set $A$, if $A$ does not have an ordering isomorphic to $\langle\mathbb{N},<\rangle$, then $A$ is a different size to $\mathbb{N}$.

This no doubt seems very plausible to many readers. And it is tempting to argue for it as follows: suppose that $A$ does not have an ordering isomorphic to $\langle\mathbb{N},\langle\rangle$; this must surely be explained by something about $A$; but the only thing relevant would seem to be $A$ 's size, i.e. $A$ must have either too few, or too many, members for them to be ordered in this way; but then (3) must hold.

\footnotetext{
${ }^{17}$ Note that the suggestion is not that we need SIZE $\rightarrow$ Function to explain the non-existence of a bijection on the basis of a difference in size. It is rather that the universal existence of such explanations would itself ensure the truth of this principle.
} 
Indeed, given how plausible these lines of thought can seem to us, it is surely likely that they are at least among the reasons that we find principles such as SizE $\rightarrow$ FunCTION and (3) so appealing.

\subsection{An Alternative Explanation}

They seem to be mistaken, however. For in the paradigm cases of infinite sets that we know not to have bijections between them, there are in fact alternative explanations that are available. To illustrate, consider $\mathbb{N}$ and $\mathcal{P}(\mathbb{N})$, and consider the following principle.

DiAgOnAL

For any $f: \mathbb{N} \rightarrow \mathcal{P}(\mathbb{N})$, there is $\quad D_{f} \in \mathcal{P}(\mathbb{N})$ such that $D_{f}=\{n \in \mathbb{N}: n \notin f(n)\}$.

This is of course familiar. But it seems to enable a sizeless explanation of the fact that there is no bijection from $\mathbb{N}$ to $\mathcal{P}(\mathbb{N})$, as follows. Let $f: \mathbb{N} \rightarrow \mathcal{P}(\mathbb{N})$, and consider $D_{f}$ as in Diagonal. This must be distinct from $f(0)$ : since by the definition of $D_{f}, 0 \in D_{f}$ iff $0 \notin f(0)$. But for the same sort of reason it must be distinct from $f(1), f(2)$ and so on. So $f$ cannot be a bijection. The gist of this explanation is simply this: given any way $f$ of assigning sets of numbers to numbers, there is a corresponding way $D_{f}$ of forming a set of numbers; and the relationship of this set to the assignment guarantees that it will be distinct from every set involved in that assignment.

Again, this sort of reasoning is of course familiar (it corresponds to a standard proof of Cantor's theorem). But the point that I want to make here is that it seems to amount to an alternative, sizeless explanation of the fact that there is no bijection from $\mathbb{N}$ to $\mathcal{P}(\mathbb{N})$. For if we are asked, 'Why is there is no such bijection?', then what I have just given would seem to constitute a completely satisfying answer. - A retort of 'I still don't understand why there is no such function' would seem totally out of place. Thus, we seem to have an explanation of why there is no such function that is quite different from (E1), i.e. as follows. (Call this (E2).)

There is no bijection from $\mathbb{N}$ to $\mathcal{P}(\mathbb{N})$

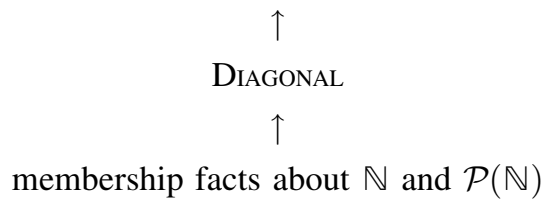

But if that is right then the proposed argument for SizE $\rightarrow$ Function fails (since that required that whenever there is no bijection from $A$ to $B$, the only possible explanation is that $A$ is a different size to $B$ ).

Perhaps, however, there will be resistance to the idea that (E2) is a genuine explanation. If so, then this might be because (E2) can-at first glance!-appear uncomfortably similar to the following 'alternative explanation' in the original case of $C$ and $D$. (Call this (E3).) 
There is no bijection from $C$ to $D$

for any $f: C \rightarrow D$, there is $x \in D$ such that for all $y \in C, f(y) \neq x$

$$
\text { membership facts about } C \text { and } D
$$

But it seems absurd to suggest that this is a genuine explanation of why there is no bijection from $C$ to $D$-and so one might worry that it is similarly absurd to claim that (E2) is a genuine explanation.

The similarity between (E2) and (E3) is merely superficial, however. For (E3) is certainly not an adequate answer to 'Why is there no bijection from $C$ to $D$ ?' Rather, it just invites the follow-up 'But why does the middle fact hold?' (i.e. why is it that for every $f: C \rightarrow D$ there is some member of $D$ that is outside the range of $f$ ?). And the only way of answering this would seem to be by saying something about size, i.e. 'Because $C$ is smaller than $D$ '.

In contrast, (E2) does, as I have noted, seem to be an adequate answer to 'Why is there no bijection from $\mathbb{N}$ to $\mathcal{P}(\mathbb{N})$ ?' It doesn't, that is, just invite a comparable follow-up. Further, although we certainly could say more about why the middle fact of (E2) holds, the natural way of doing this, unlike in the case of (E3), would make no mention of size. Rather, it would invoke the following.

(4) For any property $P$, there is a set $A=\{n \in \mathbb{N}: n$ has $P\}$.

(5) $\mathcal{P}(\mathbb{N})$ contains every set of numbers.

For these seem to enable a satisfying answer to 'Why does Diagonal hold?' (Since, for any $f: \mathbb{N} \rightarrow \mathcal{P}(\mathbb{N})$, there will be a property that applies precisely to those numbers that do not belong to their image under $f$.) Could one insist that one of (4) and (5) holds because of something about the sizes of $\mathbb{N}$ and $\mathcal{P}(\mathbb{N})$ ? It doesn't seem so: (5) is simply a statement of what $\mathcal{P}(\mathbb{N})$ is; and (4) is close to a basic fact about sets; insofar as one can give an explanation of it at all, this will amount to showing how it follows from our basic conception of set (i.e. the iterative conception), but such an explanation would make no mention of size. Thus, the similarity between (E2) and (E3) seems merely to be superficial, and so (E2) appears to be a genuine explanation.

Although the main concern of the paper is the epistemic status of SizE $\rightarrow$ FunCTION (and thus of the claim that there are different sizes of infinity), we have arrived at some metaphysical conclusions. Specifically, a picture of why certain fundamental claims about sets and functions are true. The initial strokes of this are as follows. In the case of simple pairs of finite sets, such as $C$ and $D$, if these do not have bijections between them, then the only possible explanation would indeed seem to be that they differ in size. In the case of certain infinite sets, however, such as $\mathbb{N}$ and $\mathcal{P}(\mathbb{N})$, there is a quite different sort of explanation (i.e. (E2)). This replaces talk of size with a principle to the effect that for any function from $\mathbb{N}$ to $\mathcal{P}(\mathbb{N})$, there is a certain member of $\mathcal{P}(\mathbb{N})$ that stands in a certain relation to it. Are these claims quite as exciting as those to the effect that there are different sizes of infinity? Perhaps not. 
But how revisionary-and surprising - they are can be seen by putting things in terms of counting.

Thus, it might seem obvious that if $A$ and $B$ are sets whose members one cannot count and arrive at the same result, then the only possible explanation is that they differ in size. (Compare: if $s$ and $t$ are sticks whose length one cannot measure and arrive at the same result, then the only possible explanation is that they differ in length.) But if the picture that I am sketching is right, then this apparently obvious claim is quite mistaken. For just as we can give a sizeless explanation of the fact that there is no bijection from $\mathbb{N}$ to $\mathcal{P}(\mathbb{N})$, so we can give one of the fact that we cannot count these sets and arrive at the same result, i.e. there is no cardinal that each set has a bijection to. (This explanation will be essentially similar to (E2).)

As I noted in Sect. 5.1, it can also seem obvious that if a set does not have an ordering isomorphic to $\langle\mathbb{N},<\rangle$, then the only possible explanation is that it is different size to $\mathbb{N}$. But it seems that this too must be wrong: for in the case of $\mathcal{P}(\mathbb{N})$, for example, we can give a quite different explanation. (Again, essentially similar to (E2).)

Now, the existence of these alternative explanations is presumably compatible with the sets in question also differing in size, i.e. with there also being explanations in terms of size (and thus with SizE $\rightarrow$ Function being true). But the contention of the paper as a whole is that we do not in fact have any reason to think that there are such additional explanations.

I should note that there is a certain respect in which things are a bit more nuanced than the above lets on. For everything that I have said about $\mathbb{N}$ and $\mathcal{P}(\mathbb{N})$ of course applies mutatis mutandis to any pair of sets of the form $A$ and $\mathcal{P}(A)$-including the case where $A$ is finite. That is, it is not just with certain infinite sets that the fact that there is no bijection can be explained without appealing to size. Rather, this is also true of certain finite ones. But this might set off alarm bells: does this mean that I am committed to denying that we know even the finite case of SizE $\rightarrow$ Function? There is no need to worry, however. For while I am committed to denying that the argument of Sect. 5.1 works even in the (full) finite case, it certainly doesn't follow from this that we do not know this case. For, as I will explain in Sect. 6, we can give a quite different argument for it (it is just that it will not extend to the general case).

\subsection{Simple Pairs of Finite Sets}

First, however, I want to say something more about simple pairs of finite sets such as $C$ and $D$. That is, pairs of sets without a bijection between them, but where this cannot be explained 'sizelessly' along the lines of (E2). For one might worry that I have conceded too much to the argument of Sect. 5.1 with respect to these. Specifically, I went along with the contention that, at least in the case of $C$ and $D$, the only possible explanation is indeed in terms of size. But one might worry that there is in fact even in this case a sizeless alternative (and thus that my characterization of the contrast between this case and that of $\mathbb{N}$ and $\mathcal{P}(\mathbb{N})$, for example, is mistaken). 
For it might be suggested that the following is an alternative explanation in the case of $C$ and $D$ : given the facts about which members $C$ and $D$ have, it follows that the only functions from $C$ to $D$ are $\{0 \rightarrow 2,1 \rightarrow 2\},\{0 \rightarrow 2,1 \rightarrow 3\}, \ldots$, $\{0 \rightarrow 4,1 \rightarrow 4\}$; but none of these are bijections; thus, there isn't a bijection from $C$ to $D$. The idea, that is, is that the following is an alternative to (E1). (Call this (E4).)

There is no bijection from $C$ to $D$

the functions from $C$ to $D$ are: $\{0 \rightarrow 2,1 \rightarrow 2\}, \ldots,\{0 \rightarrow 4,1 \rightarrow 4\}$

membership facts about $C$ and $D$

However, as with (E3), there would seem to be a clear sense in which this is not an adequate answer to 'Why is there no bijection from $C$ and $D$ ?' Rather, it just invites the response 'You may have said enough to convince me that there is no such function, but you haven't given me an understanding of why this is so'. For there seems to be some clear sense in which (E3) fails to isolate what it is about $C$ and $D$ that is responsible for there being no such function. In contrast, (E1), and (E2), do seem to yield genuine understandings of why there are no bijections between the sets in question (as I have in effect noted). Thus, (E4) does not in fact seem to be a genuine alternative to (E1), for it does not seem to be an explanation in anything like the way in which (E1) is.

For the purposes of this paper it is enough that there is this intuitive difference between (E1) and (E4), and so I will not try to give a precise account of what underlies it. But I would suggest that this is something like the following: there is a sense in which the middle fact of (E4) - i.e. this exhaustive description of the totality of functions from $C$ to $D$-already contains the information that we are trying to explain, and so it cannot in any illuminating sense explain this information, at least not in the way in which the middle fact of (E1) does.

\section{The Finite Case}

I will now consider the finite case of SizE $\rightarrow$ Function, and how we know it. For we are considering whether we know SizE $\rightarrow$ Function, and so it is natural to consider how we know this special case-assuming that we do-in the hope that we might be know the general one in a similar way. More pointedly, as I noted in the last section, what I said there about there being a sizeless explanation of the fact that there is no bijection between $\mathbb{N}$ and $\mathcal{P}(\mathbb{N})$ would seem to apply just as well to any other pair of sets of the form $A$ and $\mathcal{P}(A)$, including the case where $A$ is finite. So one might worry that I will be forced to deny that we know even the finite case of the principle. In this section, however, we will see that we can give a straightforward argument for this case, it is just that it will not extend to the general one. 
Specifically, we can establish the finite case of SizE $\rightarrow$ Function using just the following principles (together with something that one can prove in ZFC).

(6) For any sets $X$ and $Y$, if $X$ is finite and $X$ is a proper subset of $Y$, then $X$ is not the same size as $Y$.

(7) For any finite sets $X$ and $Y$, if there is a bijection from $X$ to $Y$ then $X$ is the same size as $Y$.

(8) $\langle z$ is the same size as $w\rangle$ is an equivalence relation.

For ZFC proves that for any sets $X$ and $Y$, either there is an injection from $X$ to $Y$, or vice versa. Thus, suppose that $A$ and $B$ are finite sets of the same size. And suppose without loss of generality that there is an injection from $A$ to $B$. That is, there is a bijection from $A$ to some $E \subseteq B$. If $E=B$ then we are done. So suppose not. Then by (6) $E$ is not the same size as $B$. But by (7) $A$ is the same size as $E$, which is impossible: by (8) together with the hypothesis that $A$ is the same size as $B$. So the finite case of SIZE $\rightarrow$ FunCTION holds.

But this argument cannot of course be extended to the general case: because (6) is not true of infinite sets.

\section{Basicness}

So we find ourselves in the following situation: we have a widely accepted, foundational principle that we seem unable to produce a convincing argument for. (At least, the initially most promising strategies seem to fail. I will however consider some more in Sect. 8.) In this sort of case, it is natural to wonder if the principle in question might not be sufficiently obvious that we are entitled to treat it as basic, i.e. believe it even in the absence of a supporting argument. After all, there surely are mathematical claims that we are entitled to so treat (and for just this reason): e.g. the axiom of extensionality in set theory, or the claim that 0 is not the successor of any natural number. Why not SizE $\rightarrow$ Function?

Well, just because it isn't in fact obvious. This seems to emerge clearly from the considerations of Sect. 5.2 in particular. For SizE $\rightarrow$ Function states that whenever $A$ is the same size as $B$, there is a bijection between them. But we saw in Sect. 5 that in the case of at least some pairs of infinite sets, the fact that there is no bijection between them has an explanation that does not involve size. But then it is surely at least prima facie possible that these are the only explanations for why there are no such functions, i.e. that it is not also the case that the sets differ in size. Indeed, it is prima facie possible that this is true of every pair of infinite sets without a bijection between them. That is, that the only explanations of these facts are along the lines of (E2), and the sets do not also differ in size. Of course, I am certainly not claiming to have established that this is actually the case (I am not even claiming to have established that $\mathbb{N}$ and $\mathcal{P}(\mathbb{N})$ are the same size). But it seems hard to maintain that there is anything obviously wrong with this picture, and so hard to maintain that $\mathrm{SizE} \rightarrow$ FunCTION is obviously true. 


\section{Inductive Arguments}

In this section I will consider the possibility of giving an inductive argument for Size $\rightarrow$ Function. That is, an argument based on the virtues of the results that it lets us derive. For although some axioms of ZFC, for example, are sufficiently obvious that we seem entitled to treat them as basic, there are others that we might seem entitled to believe only on the basis of such an argument; most notably, the axiom of replacement. What are the prospects of arguing for SIZE $\rightarrow$ FunCTION in this way?

The initial obstacle is that-unlike the axiom of replacement-SizE $\rightarrow$ FunCTION plays no role whatsoever in any proofs of mathematical results. It is of course crucial to our understanding of the significance of many such results. But it can hardly be argued for on the basis of the results that it lets us derive, for there are no such.

That leaves the option of an argument based on the non-mathematical, e.g. philosophical, results that it allows us to derive. But what might these be? One initially natural suggestion is this: ${ }^{18}$ the standard account of size (i.e. SizE $\leftrightarrow$ FUNCTION together with claims about when one set is smaller or larger than another) lets us use facts about functions - which set theory has a wealth of - to derive facts about size. For example, we know that for any sets $A$ and $B$ exactly one of the following holds: (i) there is an injection from $A$ to $B$ but not vice versa; (ii) there is a bijection from $A$ to $B$; or (iii) there is an injection from $B$ to $A$ but not vice versa. Given the standard account of size, we then get that exactly one of the following holds: (I) $A$ is smaller than $B$; (II) $A$ is the same size as $B$; or (III) $A$ is larger than $B$. This is a nice result, and so one might suggest that it provides the basis for an inductive argument for the standard account (which of course includes SIZE $\rightarrow$ FunCTION).

The problem with this suggestion, however, is that although the standard account does indeed allow us to derive this, that is also true of its most plausible alternative, i.e. that on which all infinite sets are the same size. (It is easy to derive from this that exactly one of (I-III) will always hold.) More generally, it is hard to see how anything like this suggestion is going to succeed, given that the 'one (infinite) size' account would seem to yield a theory of size that is simpler and more powerful than that yielded by the standard account: more powerful because while the standard account leaves us with many questions about infinite size that we cannot answer, even with the full force of ZFC (e.g. 'Is there a size between those of $\mathbb{N}$ and $\mathcal{P}(\mathbb{N})$ ?'), the one size account allows us to answer all such questions. It seems unlikely, then, that we are going to be able to establish SizE $\rightarrow$ Function on the basis of such an inductive argument.

\section{9 'Size-Like' Properties (Again)}

Thus it appears that we are not in a position to know Size $\rightarrow$ Function. But what about SizE* $\rightarrow$ FunCtion? In Sect. 4 I argued against the idea that this is just obvious. But we are now in a position to say something stronger. For the above arguments against the claim that we are in a position know SizE $\rightarrow$ Function would seem to

18 Thanks here to Phillip Bricker and Jeremy Goodman. 
apply just as well to the weaker one that we are in a position to know SiZE $\rightarrow$ FunCTION.

For example, we saw in Sect. 5 that in the paradigm cases of infinite sets without bijections between them, we can explain this without appealing to size. But it is not simply that the alternative explanation does not involve size-neither does it involve any 'size-like' property, i.e. any property that is similar to size. For it is not as if we replaced the claim that $\mathbb{N}$ and $\mathcal{P}(\mathbb{N})$ differ in size with one to the effect that they differ in size*, for some such property size*. Rather, we replaced this claim with Diagonal, which is of a completely different form, and which does not involve anything like sizes.

Further, similar points can be made about each of the other arguments. We thus seem no more in a position to know SizE $\rightarrow$ Function than we are to know Size $\rightarrow$ FunCtion.

\section{Conclusion}

I hope, then, to have made a plausible case to the effect that we are not in a position to know SizE $\rightarrow$ Function. In conclusion I will mention some directions that future work on these issues might take.

The most obvious question that is raised-or reopened-by the above is of course: are there, in fact, different sizes of infinity? How might one go about investigating this? A natural strategy would seem to be to spend a bit more time thinking about what sizes actually are, rather than focusing simply on a certain criterion that we know tracks sameness of size in a range of cases.

But another, to a great extent independent, direction would add to the picture that I sketched in Sect. 5.2. According to this, a number of claims about sets and functions that one might have thought can only be explained in terms of size, can in fact be explained in quite different ways. In particular, we looked at a number of examples involving $\mathbb{N}$ and $\mathcal{P}(\mathbb{N})$. But it is natural to ask how far these ideas can be taken. What I said about $\mathbb{N}$ and $\mathcal{P}(\mathbb{N})$ would seem to extend straightforwardly enough to other standard examples of sets that we know not to have bijections between them. But can a similar claim be made about every pair of infinite sets without such a function between them? What about other set-theoretic claims (i.e. other claims expressible in the language of set theory)? Can each such truth be explained in a way that does not involve differences in infinite size? It is natural to ask about the relation of such questions to the Löwenheim-Skolem theorems. That is, the relation between, on the one hand, explanations of set-theoretic claims that do not involve differences in infinite size, and, on the other, models of such claims that do not in fact contain such differences. These, then, are at least some of the questions that I hope to pursue in future work.

Acknowledgements I would like to thank Mark Balaguer, Phillip Bricker, Michaela McSweeney, Agustín Rayo, Marcus Rossberg, Ian Rumfitt, Joshua Schechter, audiences at the Oxford Philosophy of Mathematics Conference and the Vancouver Summer Philosophy Conference, and two referees for this journal. 
Open Access This article is distributed under the terms of the Creative Commons Attribution 4.0 International License (http://creativecommons.org/licenses/by/4.0/), which permits unrestricted use, distribution, and reproduction in any medium, provided you give appropriate credit to the original author(s) and the source, provide a link to the Creative Commons license, and indicate if changes were made.

\section{References}

Benacerraf, P. (1965). What numbers could not be. Philosophical Review, 74, 47-73.

Bolzano, B. (1851). Paradoxes of the infinite (D. A. Steele, 1950, Trans.). New Haven: Yale University Press.

Gödel, K. (1964). What is Cantor's continuum problem? In S. Feferman et al. (Eds) Collected works: Volume II, Publications 1938-1974 (1990, pp. 254-270). Oxford: Oxford University Press.

Hale, B., \& Wright, C. (2001). The reason's proper study: Essays towards a neo-Fregean philosophy of mathematics. Oxford: Clarendon Press.

Hallett, M. (1984). Cantorian set theory and limitation of size. Oxford: Clarendon Press.

Mancosu, P. (2009). Measuring the size of infinite collections of natural numbers: Was Cantor's theory of the infinite inevitable? Review of Symbolic Logic, 2, 612-646.

Parker, M. (2009). Philosophical method and Galileo's paradox of infinity. In B. van Kerkove (Ed.), New perspectives on mathematical practices (pp. 76-113). Hackensack: World Scientific.

Rayo, A. (2013). The construction of logical space. Oxford: Oxford University Press.

Whittle, B. (2015). On infinite size. In Oxford studies in metaphysics: Volume 9 (pp. 3-19). Oxford: Oxford University Press.

Wright, C. (1983). Frege's conception of numbers as objects. Aberdeen: Aberdeen University Press.

Wright, C. (1997). On the philosophical significance of Frege's theorem. In Hale and Wright (2001) (pp. 272-306). 